\title{
Positive solutions to $p$-Laplacian fractional differential equations with infinite-point boundary value conditions
}

\author{
Han Wang ${ }^{1}$, Suli Liu ${ }^{1}$ and Huilai Li ${ }^{1 *}$ (D)
}

"Correspondence:

lihuilai@jlu.edu.cn

'Department of Mathematics, Jilin

University, Changchun, P.R. China

\section{Springer}

\begin{abstract}
This paper is devoted to an investigation of the multiple positive solutions to a class of infinite-point boundary value problems of nonlinear fractional differential equations coupled with the p-Laplacian operators and infinite-point boundary value conditions. By means of the properties of Green's function and fixed point theorems, we establish the suitable criteria to guarantee the existence of positive solutions. Finally, an example is given in order to illustrate the main results.
\end{abstract}

Keywords: Fractional differential equations; Infinite-point boundary conditions; p-Laplacian operators; Fixed point theorems

\section{Introduction}

With the advance of technology, researchers are not satisfied with the limitations of the integer order calculus anymore; therefore, fractional calculus - the expansion and extension of the integer one-is brought into the public. There is no doubt the study on nonlinear fractional differential equations has become an issue of focus and is widely being used in many fields, such as physics [1, 2], biology [3], energy [4], chemical engineering [5], pharmacokinetics [6].

Based on practical application, researchers established lots of fractional differential equation models, so how to get the solutions to the equations is a big challenge to them. As is well known, it is difficult to give the exact solution, so giving the properties of the solutions is a significant point.

A great number of researchers are focused on the existence of solutions for linear or nonlinear FODE with different boundary conditions. Take Zhang [7] for example, one studied the existence of minimal non-negative solution for a class of nonlinear fractional integrodifferential equations by applying the cone theory and the monotone iterative technique:

$$
\left\{\begin{array}{l}
D_{0^{+}}^{\alpha} x(t)+f(t, x(t), T x(t), S x(t))=0, \quad t \in[0, \infty), \\
x(0)=0, \quad D_{0^{+}}^{\alpha-1} x(\infty)=x^{*}
\end{array}\right.
$$

where

$$
(T x)(t)=\int_{0}^{t} k(t, s) x(s) d s, \quad(S x)(t)=\int_{0}^{\infty} h(t, s) x(s) d s,
$$

(c) The Author(s) 2018. This article is distributed under the terms of the Creative Commons Attribution 4.0 International License (http://creativecommons.org/licenses/by/4.0/), which permits unrestricted use, distribution, and reproduction in any medium, provided you give appropriate credit to the original author(s) and the source, provide a link to the Creative Commons license, and indicate if changes were made. 
and $1<\alpha \leq 2, D_{0^{+}}^{\alpha}$ is the Riemann-Liouville derivative, $f, k, h$ are continuous functions.

Wang [8] considered the iterative positive solutions for a class of nonlocal fractional differential equations with nonlocal Hadamard integral and discrete boundary conditions by the monotone iterative method:

$$
\left\{\begin{array}{l}
{ }^{H} D^{\alpha} x(t)+\delta(t) f(t, x(t))=0, \quad t \in(1, \infty) \\
x(1)=x^{\prime}(1)=0 \\
{ }^{H} D^{\alpha-1} x(\infty)=a^{H} I^{\beta} x(\xi)+b \sum_{i=1}^{m-2} c_{i} x\left(\eta_{i}\right)
\end{array}\right.
$$

where $2<\alpha \leq 3, a, b$ are real constants, $c_{i}(i=1,2, \ldots, m-2)$ are positive real constants, $1<\xi<\eta_{1}<\eta_{2}<\cdots<\eta_{m-2}<+\infty$, and ${ }^{H} D^{\alpha}$ is the Hadamard fractional derivative.

As for Guo [9], they investigated the existence of solutions for the following infinitepoint BVP with Caputo fractional derivative by Avery-Peterson's fixed point theorem:

$$
\left\{\begin{array}{l}
{ }^{c} D^{\alpha} x(t)+f\left(t, x(t), x^{\prime}(t)\right)=0, \quad 0<t<1, \\
x(0)=x^{\prime \prime}(0)=0, \quad x^{\prime}(1)=\sum_{j=1}^{\infty} \zeta_{j} x\left(\xi_{j}\right),
\end{array}\right.
$$

where $2<\alpha \leq 3, \zeta_{j} \geq 0,0<\xi_{1}<\xi_{2}<\cdots<\xi_{i}<\cdots<1$, and $\sum_{i=1}^{\infty} \zeta_{i} \xi_{i}<1$.

Recently, some scientists have become interested in the study of the fractional differential equations with p-Laplacian operators [10-13]. For example, Xiping Liu [12, 13] obtained some new results on the existence of positive solutions for the four-point BVP with mixed fractional derivatives and p-Laplacian operator by a new method of lower and upper solutions which is based on the monotone iterative technique:

$$
\left\{\begin{array}{l}
D_{0^{+}}^{\beta}\left(\phi_{p}\left({ }^{C} D_{0^{+}}^{\alpha} x(t)\right)\right)=f\left(t, x(t),{ }^{C} D_{0^{+}}^{\alpha} x(t)\right), \quad t \in(0,1), \\
{ }^{c} D_{0^{+}}^{\alpha} x(0)=x^{\prime}(0)=0, \\
x(1)=r_{1} x(\eta), \quad{ }^{C} D_{0^{+}}^{\alpha} x(1)=r_{2}^{C} D_{0^{+}}^{\alpha} x(\xi),
\end{array}\right.
$$

where $1<\alpha, \beta \leq 2, r_{1}, r_{2} \geq 0, f \in C([0,1] \times[0,+\infty) \times(-\infty, 0],[0,+\infty)),{ }^{c} D_{0^{+}}^{\alpha}$ is the Caputo derivative, $D_{0^{+}}^{\alpha}$ is the Riemann-Liouville derivative, $\phi_{p}$ is the p-Laplacian operator which is defined as $\phi_{p}(s)=|s|^{p-2} s(p>1), \phi_{q}$ is the inverse function of $\phi_{p}$ and $\frac{1}{p}+\frac{1}{q}=1$.

In this paper, we consider a class of nonlinear fractional differential equations coupled with the p-Laplacian operator and infinite-point boundary value conditions:

$$
\left\{\begin{array}{l}
-D_{0^{+}}^{\beta}\left(\phi_{p}\left(D_{0^{+}}^{\alpha} x(t)-\lambda x(t)\right)\right)=f(t, x(t)), \quad t \in(0,1], \\
\lim _{t \rightarrow 0^{+}} t^{1-\alpha} x(t)=\sum_{i=1}^{\infty} \mu_{i} x\left(\xi_{i}\right), \\
\lim _{t \rightarrow 0^{+}} t^{2-\beta}\left(\phi_{p}\left(D_{0^{+}}^{\alpha} x(t)-\lambda x(t)\right)\right)=\phi_{p}\left(D_{0^{+}}^{\alpha} x(1)-\lambda x(1)\right)=0,
\end{array}\right.
$$

where $0<\alpha \leq 1,1<\beta \leq 2, \lambda<0, \mu_{i} \geq 0,0<\xi_{i}<1, i \in \mathbb{R}^{+}, \sum_{i=1}^{\infty} \mu_{i} \xi_{i}^{\alpha-1}<1, f \in C([0,1] \times$ $[0,+\infty),[0,+\infty)), \phi_{p}$ is the p-Laplacian operator and $D^{\alpha}, D^{\beta}$ are the Riemann-Liouville fractional derivatives.

To the best of our knowledge, few researchers studied fractional differential equations with p-Laplacian by the Riemann-Liouville derivative; this condition coupled with values 
at infinite number of points is not covered in the previous situations, and it is more general for the fractional differential equation models. By means of the properties of Green's function, Krasnosel'skii fixed point theorem, the corollary of Krasnosel'skii fixed point theorem and Avery-Peterson fixed point theorem, we establish the suitable criteria to warrant the existence of the positive solutions for the BVP (1).

The rest of the paper is organized as follows. In Sect. 2, we list several basic definitions and lemmas to prepare for the further study in this paper. On top of that, the main result is showed in Sect. 3. In Sect. 4, we give an example to illustrate our main results.

\section{Preliminaries}

As is well known, $C[a, b]$ is the Banach space of all continuous real functions defined on $[a, b]$ with the norm $\|x\|=\sup _{t \in[a, b]}|x(t)|$ and $L^{1}[a, b]$ is for the Lebesgue integrable real functions defined on $[a, b]$. In this paper, we consider the following space:

$$
C_{1-\alpha}[0,1]=\left\{x(t): t^{1-\alpha} x(t) \in C[0,1], 0<\alpha \leq 1\right\},
$$

endowed with its norm $\|x\|_{1-\alpha}=\sup _{t \in[0,1]} t^{1-\alpha}|x(t)|$. Obviously, $C_{1-\alpha}[0,1]$ is also a Banach space; moreover, $C_{1-\alpha}[0,1] \subset L^{1}[0,1]$.

Definition 2.1 ([14]) Let $\alpha>0$, the Riemann-Liouville fractional integral of the function $f$ is defined by

$$
I_{0^{+}}^{\alpha} f(t)=\frac{1}{\Gamma(\alpha)} \int_{0}^{t}(t-s)^{\alpha-1} f(s) d s .
$$

Definition 2.2 ([14]) Let $\alpha>0$, the Riemann-Liouville fractional derivative of the function $f$ is defined by

$$
D_{0^{+}}^{\alpha} f(t)=\frac{1}{\Gamma(n-\alpha)}\left(\frac{d}{d t}\right)^{n} \int_{0}^{t}(t-s)^{n-\alpha-1} f(s) d s,
$$

where $n$ is the smallest integer greater than or equal to $\alpha$.

Definition 2.3 ([15]) The two-parameter Mittag-Leffler function is defined by

$$
E_{\alpha, \beta}(\zeta)=\sum_{k=0}^{\infty} \frac{\zeta^{k}}{\Gamma(\alpha k+\beta)}, \quad \alpha, \beta>0, \zeta \in \mathbb{R}
$$

Definition 2.4 ([16]) If the map $T$ satisfies

$$
T(a x+(1-a) y) \leq(\geq) a T(x)+(1-a) T(y), \quad x, y \in P, a \in[0,1],
$$

then $T$ is called a non-negative convex (concave) operator on a cone $P$.

Lemma 2.1 ([17]) Assume $\alpha>0, u \in C(0,1) \cap L(0,1)$, then

$$
I_{0^{+}}^{\alpha} D_{0^{+}}^{\alpha} f(t)=u(t)+c_{1} t^{\alpha-1}+c_{2} t^{\alpha-2}+\cdots+c_{n} t^{\alpha-n}
$$

where $c_{i} \in \mathbb{R}, i=1,2, \ldots, n$ with $n$ is the smallest integer greater than or equal to $\alpha$. 
Lemma 2.2 ([18]) Assume $\alpha \in(0,1], \beta>0, \lambda$ is a negative constant, then the following conclusions hold:

(i) $\lim _{t \rightarrow 0^{+}} E_{\alpha, \beta}\left(\lambda t^{\alpha}\right)=\frac{1}{\Gamma(\beta)}$,

(ii) $E_{\alpha, \alpha}\left(\lambda t^{\alpha}\right)>0$ for $\forall t \in[0,+\infty)$,

(iii) $E_{\alpha, \alpha}\left(\lambda t^{\alpha}\right)$ is decreasing in $t$ for $\forall t \in(0,+\infty)$,

(iv) $E_{\alpha, \alpha}\left(\lambda t^{\alpha}\right) \leq E_{\alpha, \alpha+1}\left(\lambda t^{\alpha}\right)$ for $\forall t \in(0,+\infty)$.

Lemma 2.3 Assume $\sigma(t) \in C(0,1], 0<\alpha \leq 1$, then the problem

$$
\left\{\begin{array}{l}
D_{0+}^{\alpha} x(t)-\lambda x(t)=\sigma(t), \quad t \in(0,1], \\
\lim _{t \rightarrow 0^{+}} t^{1-\alpha} x(t)=\sum_{i=1}^{\infty} \mu_{i} x\left(\xi_{i}\right),
\end{array}\right.
$$

has an unique solution

$$
x(t)=\int_{0}^{1} G(t, s) \sigma(s) d s
$$

where

$$
G(t, s)=\left\{\begin{array}{l}
\frac{\Gamma(\alpha) E_{\alpha, \alpha}\left(\lambda t^{\alpha}\right) \sum_{s \leq \xi_{i}} \mu_{i}\left(\xi_{i}-s\right)^{\alpha-1} E_{\alpha, \alpha}\left(\lambda\left(\xi_{i}-s\right)^{\alpha}\right)}{t^{1-\alpha}\left(1-\Gamma(\alpha) \sum_{i=1}^{\infty} \mu_{i} \xi_{i}^{\alpha-1} E_{\alpha, \alpha}\left(\lambda \xi_{i}^{\alpha}\right)\right)}+\frac{E_{\alpha, \alpha}\left(\lambda(t-\alpha)^{\alpha}\right)}{(t-s)^{1-\alpha}} \\
0 \leq s<t \leq 1 \\
\frac{\Gamma(\alpha) E_{\alpha, \alpha}\left(\lambda t^{\alpha}\right) \sum_{s \leq \xi_{\xi}} \mu_{i}\left(\xi_{i}-s\right)^{\alpha-1} E_{\alpha, \alpha}\left(\lambda\left(\xi_{i}-s\right)^{\alpha}\right)}{t^{1-\alpha}\left(1-\Gamma(\alpha) \sum_{i=1}^{\infty} \mu_{i} \xi_{i}^{\alpha-1} E_{\alpha, \alpha}\left(\lambda \xi_{i}^{\alpha}\right)\right)} \\
0<t \leq s \leq 1 .
\end{array}\right.
$$

Proof According to ([19]), if $\lim _{t \rightarrow 0^{+}} t^{(1-\alpha)} x(t)=c_{0}$, we can easily get

$$
x(t)=c_{0} \Gamma(\alpha) t^{\alpha-1} E_{\alpha, \alpha}\left(\lambda t^{\alpha}\right)+\int_{0}^{t}(t-s)^{\alpha-1} E_{\alpha, \alpha}\left(\lambda(t-\alpha)^{\alpha}\right) \sigma(s) d s .
$$

In particular, if we choose $c_{0}=\sum_{i=1}^{\infty} \mu_{i} x\left(\xi_{i}\right)$, by simple calculation,

$$
c_{0}=\frac{\sum_{i=1}^{\infty} \mu_{i} \int_{0}^{\xi_{i}}\left(\xi_{i}-s\right)^{\alpha-1} E_{\alpha, \alpha}\left(\lambda\left(\xi_{i}-s\right)^{\alpha}\right) \sigma(s) d s}{1-\Gamma(\alpha) \sum_{i=1}^{\infty} \mu_{i} \xi_{i}^{\alpha-1} E_{\alpha, \alpha}\left(\lambda \xi_{i}^{\alpha}\right)} .
$$

Hence,

$$
\begin{aligned}
x(t)= & \frac{\Gamma(\alpha) t^{\alpha-1}}{1-\Gamma(\alpha) \sum_{i=1}^{\infty} \mu_{i} \xi_{i}^{\alpha-1} E_{\alpha, \alpha}\left(\lambda \xi_{i}^{\alpha}\right)} \\
& \times \sum_{i=1}^{\infty} \mu_{i} E_{\alpha, \alpha}\left(\lambda t^{\alpha}\right) \int_{0}^{\xi_{i}}\left(\xi_{i}-s\right)^{\alpha-1} E_{\alpha, \alpha}\left(\lambda\left(\xi_{i}-s\right)^{\alpha}\right) \sigma(s) d s \\
& +\int_{0}^{t}(t-s)^{\alpha-1} E_{\alpha, \alpha}\left(\lambda(t-\alpha)^{\alpha}\right) \sigma(s) d s \\
= & \int_{0}^{1} \frac{\Gamma(\alpha) E_{\alpha, \alpha}\left(\lambda t^{\alpha}\right) \sum_{s \leq \xi_{i}} \mu_{i}\left(\xi_{i}-s\right)^{\alpha-1} E_{\alpha, \alpha}\left(\lambda\left(\xi_{i}-s\right)^{\alpha}\right)}{t^{1-\alpha}\left(1-\Gamma(\alpha) \sum_{i=1}^{\infty} \mu_{i} \xi_{i}^{\alpha-1} E_{\alpha, \alpha}\left(\lambda \xi_{i}^{\alpha}\right)\right)} \sigma(s) d s \\
& +\int_{0}^{t} \frac{E_{\alpha, \alpha}\left(\lambda(t-\alpha)^{\alpha}\right)}{(t-s)^{1-\alpha}} \sigma(s) d s
\end{aligned}
$$




$$
=\int_{0}^{1} G(t, s) \sigma(s) d s
$$

where $G(t, s)$ is given by (4).

The proof is finished.

Lemma 2.4 Assume $\alpha \in(0,1], \lambda$ is a negative constant, then

(i) $G(t, s)>0$ for $t \in(0,1], s \in[0,1)$,

(ii) $t^{1-\alpha} G(t, s) \geq \frac{\Gamma(\alpha)\left(E_{\alpha, \alpha}(\lambda)\right)^{2} \sum_{s \leq \xi_{i}} \mu_{i} \xi_{i}^{\alpha-1}}{1-\Gamma(\alpha) \sum_{i=1}^{\infty} \mu_{i} \xi_{i}^{\alpha-1} E_{\alpha, \alpha}(\lambda)} \triangleq m$ for $t, s \in[0,1]$,

(iii) $\min _{t \in[0,1]} t^{1-\alpha} G(t, s) \geq \rho \max _{t \in[0,1]} t^{1-\alpha} G(t, s)$ for $\rho \in(0,1)$,

(iv) $t^{1-\alpha} \int_{0}^{1} G(t, s) d s \leq\left(\Gamma(\alpha)\left(1-\sum_{i=0}^{\infty} \mu_{i} \xi_{i}^{\alpha-1}\right)\right)^{-1} \triangleq n$ for $t, s \in[0,1]$.

Proof (i) By simple calculation,

$$
1-\Gamma(\alpha) \sum_{i=1}^{\infty} \mu_{i} \xi_{i}^{\alpha-1} E_{\alpha, \alpha}\left(\lambda \xi_{i}^{\alpha}\right) \geq 1-\sum_{i=1}^{\infty} \mu_{i} \xi_{i}^{\alpha-1}>0
$$

In addition, according to Lemma 2.2, we can easily see that (i) holds.

(ii) Since $(t-s)^{\alpha-1} E_{\alpha, \alpha}\left(\lambda(t-s)^{\alpha}\right) \geq 0$ for all $0 \leq s<t \leq 1$,

$$
\begin{aligned}
t^{1-\alpha} G(t, s) & \geq \frac{\Gamma(\alpha) E_{\alpha, \alpha}\left(\lambda t^{\alpha}\right) \sum_{s \leq \xi_{i}} \mu_{i}\left(\xi_{i}-s\right)^{\alpha-1} E_{\alpha, \alpha}\left(\lambda\left(\xi_{i}-s\right)^{\alpha}\right)}{1-\Gamma(\alpha) \sum_{i=1}^{\infty} \mu_{i} \xi_{i}^{\alpha-1} E_{\alpha, \alpha}\left(\lambda \xi_{i}^{\alpha}\right)} \\
& \geq \frac{\Gamma(\alpha)\left(E_{\alpha, \alpha}(\lambda)\right)^{2} \sum_{s \leq \xi_{i}} \mu_{i} \xi_{i}^{\alpha-1}}{1-\Gamma(\alpha) E_{\alpha, \alpha}(\lambda) \sum_{i=1}^{\infty} \mu_{i} \xi_{i}^{\alpha-1}} \\
& \triangleq m>0 .
\end{aligned}
$$

(iii) As $t^{1-\alpha} G(t, s)$ is increasing in $s$ and decreasing in $t$

$$
\max _{t \in[0,1]} t^{1-\alpha} G(t, s)=\frac{\sum_{s \leq \xi_{i}} \mu_{i}\left(\xi_{i}-s\right)^{\alpha-1} E_{\alpha, \alpha}\left(\lambda\left(\xi_{i}-s\right)^{\alpha}\right)}{1-\Gamma(\alpha) \sum_{i=1}^{\infty} \mu_{i} \xi_{i}^{\alpha-1} E_{\alpha, \alpha}\left(\lambda \xi_{i}^{\alpha}\right)}
$$

According to Lemma 2.4(i), we have

$$
\begin{aligned}
\min _{t \in[0,1]} t^{1-\alpha} G(t, s) & \geq \Gamma(\alpha) E_{\alpha, \alpha}(\lambda) \max _{t \in[0,1]} t^{1-\alpha} G(t, s) \\
& \triangleq \rho \max _{t \in[0,1]} t^{1-\alpha} G(t, s)
\end{aligned}
$$

where $\rho=\Gamma(\alpha) E_{\alpha, \alpha}(\lambda)$, so $0<\rho<1$.

(iv) We divide this proof into two parts:

Part 1: For $0 \leq s \leq t \leq 1$,

$$
\begin{aligned}
t^{1-\alpha} \int_{0}^{1} G(t, s) d s= & \int_{0}^{1} \frac{\Gamma(\alpha) E_{\alpha, \alpha}\left(\lambda t^{\alpha}\right) \sum_{s \leq \xi_{i}} \mu_{i}\left(\xi_{i}-s\right)^{\alpha-1} E_{\alpha, \alpha}\left(\lambda\left(\xi_{i}-s\right)^{\alpha}\right)}{1-\Gamma(\alpha) \sum_{i=1}^{\infty} \mu_{i} \xi_{i}^{\alpha-1} E_{\alpha, \alpha}\left(\lambda \xi_{i}^{\alpha}\right)} d s \\
& +\int_{0}^{t} t^{1-\alpha}(t-s)^{\alpha-1} E_{\alpha, \alpha}\left(\lambda(t-\alpha)^{\alpha}\right) d s \\
\leq & \frac{\Gamma(\alpha) E_{\alpha, \alpha}\left(\lambda t^{\alpha}\right) \sum_{i=1}^{\infty} \mu_{i} \xi_{i}^{\alpha-1}}{1-\Gamma(\alpha) \sum_{i=1}^{\infty} \mu_{i} \xi_{i}^{\alpha-1} E_{\alpha, \alpha}\left(\lambda \xi_{i}^{\alpha}\right)}+t E_{\alpha, \alpha+1}\left(\lambda t^{\alpha}\right)
\end{aligned}
$$




$$
\begin{aligned}
& \leq \frac{\sum_{i=1}^{\infty} \mu_{i} \xi_{i}^{\alpha-1}}{\Gamma(\alpha)\left(1-\sum_{i=1}^{\infty} \mu_{i} \xi_{i}^{\alpha-1}\right)}+\frac{1}{\Gamma(\alpha)} \\
& =\frac{1}{\Gamma(\alpha)\left(1-\sum_{i=1}^{\infty} \mu_{i} \xi_{i}^{\alpha-1}\right)} \triangleq n .
\end{aligned}
$$

Part 2: For $0 \leq t \leq s \leq 1$,

$$
\begin{aligned}
t^{1-\alpha} \int_{0}^{1} G(t, s) d s & =\int_{0}^{1} \frac{\Gamma(\alpha) E_{\alpha, \alpha}\left(\lambda t^{\alpha}\right) \sum_{s \leq \xi_{i}} \mu_{i}\left(\xi_{i}-s\right)^{\alpha-1} E_{\alpha, \alpha}\left(\lambda\left(\xi_{i}-s\right)^{\alpha}\right)}{1-\Gamma(\alpha) \sum_{i=1}^{\infty} \mu_{i} \xi_{i}^{\alpha-1} E_{\alpha, \alpha}\left(\lambda \xi_{i}^{\alpha}\right)} d s \\
& \leq \frac{\Gamma(\alpha) E_{\alpha, \alpha}\left(\lambda t^{\alpha}\right) \sum_{i=1}^{\infty} \mu_{i} \xi_{i}^{\alpha-1}}{1-\Gamma(\alpha) \sum_{i=1}^{\infty} \mu_{i} \xi_{i}^{\alpha-1} E_{\alpha, \alpha}\left(\lambda \xi_{i}^{\alpha}\right)} \\
& \leq \frac{\sum_{i=1}^{\infty} \mu_{i} \xi_{i}^{\alpha-1}}{\Gamma(\alpha)\left(1-\sum_{i=1}^{\infty} \mu_{i} \xi_{i}^{\alpha-1}\right)} \\
& <\frac{1}{\Gamma(\alpha)\left(1-\sum_{i=1}^{\infty} \mu_{i} \xi_{i}^{\alpha-1}\right)} \triangleq n .
\end{aligned}
$$

The proof is completed.

Lemma 2.5 Assume $h(t) \in C(0,1], 1<\beta \leq 2$, then the problem

$$
\left\{\begin{array}{l}
-D_{0+}^{\beta} u(t)=h(t), \quad t \in(0,1], \\
\lim _{t \rightarrow 0^{+}} t^{2-\beta} u(t)=u(1)=0,
\end{array}\right.
$$

has an unique solution

$$
u(t)=\int_{0}^{1} H(t, s) h(s) d s,
$$

where

$$
H(t, s)=\frac{1}{\Gamma(\beta)} \begin{cases}t^{\beta-1}(1-s)^{\beta-1}-(t-s)^{\beta-1}, & 0 \leq s<t \leq 1 \\ t^{\beta-1}(1-s)^{\beta-1}, & 0<t \leq s \leq 1 .\end{cases}
$$

Proof According to Lemma 2.1, we can easily get

$$
u(t)=-I_{0^{+}}^{\beta} h(t)+c_{1} t^{\beta-1}+c_{2} t^{\beta-2},
$$

by the condition (6), we obtain

$$
c_{1}=I_{0^{+}}^{\beta} h(1), \quad c_{2}=0 .
$$

Hence,

$$
u(t)=\frac{t^{\beta-1}}{\Gamma(\beta)} \int_{0}^{1}(1-s)^{\beta-1} h(s) d s-\frac{1}{\Gamma(\beta)} \int_{0}^{t}(t-s)^{\beta-1} h(s) d s=\int_{0}^{1} H(t, s) h(s) d s,
$$

where $H(t, s)$ is given by $(8)$.

The proof is finished. 
Lemma 2.6 ([20]) Assume $\beta \in(1,2]$, then

(i) $H(t, s)>0$ for $t \in(0,1], s \in[0,1)$,

(ii) $H(t, s)=H(1-s, 1-t)$ for $t, s \in(0,1)$,

(iii) $t^{\beta-1}(1-t) s(1-s)^{\beta-1} \leq \Gamma(\beta) H(t, s) \leq(\beta-1) s(1-s)^{\beta-1}$ for $t \in(0,1], s \in[0,1)$,

(iv) $t^{\beta-1}(1-t) s(1-s)^{\beta-1} \leq \Gamma(\beta) H(t, s) \leq(\beta-1)(1-t) t^{\beta-1}$ for $t \in(0,1], s \in[0,1)$.

Lemma 2.7 Suppose $h(t)=f(t, x(t)), \sigma(t)=\phi_{q}(u(t))$ hold, then we obtain the unique solution of $B V P(1)$ :

$$
x(t)=\int_{0}^{1} G(t, s) \phi_{q}\left(\int_{0}^{1} H(s, \tau) f(\tau, x(\tau)) d \tau\right) d s .
$$

\section{Main results}

In this section, we establish the related condition to warrant the existence of the multiple positive solutions for BVP (1) by means of the properties of Green's function we proved in the last section and the well-known Krasnosel'skii fixed point theorem, the corollary of Krasnosel'skii fixed point theorem and the Avery-Peterson fixed point theorem.

Let $E=\left\{x(t): x(t) \in C_{1-\alpha}[0,1]\right\}$. Define $P \subset E$,

$$
P=\left\{x(t) \in E: x(t) \geq 0, \min _{t \in[0,1]} t^{1-\alpha} x(t) \geq \rho \max _{t \in[0,1]} t^{1-\alpha} x(t)\right\},
$$

where $\rho$ is defined in Lemma 2.4.

Suppose $T: P \rightarrow E$ is the operator defined by

$$
T x(t):=\int_{0}^{1} G(t, s) \phi_{q}\left(\int_{0}^{1} H(s, \tau) f(\tau, x(\tau)) d \tau\right) d s .
$$

Throughout this section, suppose the following assumption holds:

$\left(\mathrm{H}_{0}\right)|f(t, x)| \leq M$.

Lemma 3.1 $T: P \rightarrow P$ is a completely continuous operator.

Proof Step 1: We show that $T(P) \subseteq P$.

For $\forall x \in P$, since the continuity of $G(t, s), H(t, s), f(t, x(t))$, it is easy to get $t^{1-\alpha} T x(t) \in$ $C[0,1]$. According to Lemma 2.4 and Lemma 2.6, we obtain $G(t, s), H(t, s) \geq 0$, thanks to $f(t, x(t))$ being non-negative, therefore, $T x(t) \geq 0$. Furthermore,

$$
\begin{aligned}
\min _{t \in[0,1]} t^{1-\alpha} T x(t) & =\min _{t \in[0,1]} t^{1-\alpha} \int_{0}^{1} G(t, s) \phi_{q}\left(\int_{0}^{1} H(s, \tau) f(\tau, x(\tau)) d \tau\right) d s \\
& =\int_{0}^{1} \min _{t \in[0,1]} t^{1-\alpha} G(t, s) \phi_{q}\left(\int_{0}^{1} H(s, \tau) f(\tau, x(\tau)) d \tau\right) d s \\
& \geq \int_{0}^{1} \rho \max _{t \in[0,1]} t^{1-\alpha} G(t, s) \phi_{q}\left(\int_{0}^{1} H(s, \tau) f(\tau, x(\tau)) d \tau\right) d s \\
& =\rho \max _{t \in[0,1]} T x(t) .
\end{aligned}
$$

Hence, $T(P) \subseteq P$. 
Step 2: We will prove that $T$ is uniformly bounded. We have

$$
\begin{aligned}
\|T x(t)\|_{1-\alpha} & =\left|t^{1-\alpha} \int_{0}^{1} G(t, s) \phi_{q}\left(\int_{0}^{1} H(s, \tau) f(\tau, x(\tau)) d \tau\right) d s\right| \\
& \leq \int_{0}^{1}\left|t^{1-\alpha} G(t, s)\right| \phi_{q}\left(\int_{0}^{1}|H(s, \tau)| f(\tau, x(\tau)) \mid d \tau\right) d s \\
& <n \phi_{q}\left(\frac{(\beta-1) M}{\Gamma(\beta)}\right) \\
& =n \phi_{q}\left(\frac{M}{\Gamma(\beta-1)}\right) \triangleq M_{1} .
\end{aligned}
$$

Therefore, $\|T x(t)\|_{1-\alpha}<M_{1}$, which means $T$ is uniformly bounded.

Step 3: We will give the proof of the equicontinuity of $T$.

Obviously, $t^{1-\alpha} G(t, s)$ is uniformly continuous in the interval $[0,1] \times[0,1]$. Therefore, for all $\varepsilon>0$, there exists $\delta>0$, without loss of generality, we choose $t_{1}, t_{2} \in[0,1]$, when $\left|t_{1}-t_{2}\right|<\delta$, we have

$$
\left|G\left(t_{1}, s\right)-G\left(t_{2}, s\right)\right|<\frac{\varepsilon}{\phi_{q}(M) \int_{0}^{1} \phi_{q}\left(\int_{0}^{1} H(s, \tau) d \tau\right) d s}
$$

Thus,

$$
\begin{aligned}
\left\|T x\left(t_{1}\right)-T x\left(t_{2}\right)\right\|_{1-\alpha} \\
=\mid t_{1}^{1-\alpha} \int_{0}^{1} G\left(t_{1}, s\right) \phi_{q}\left(\int_{0}^{1} H(s, \tau) f(\tau, x(\tau)) d \tau\right) d s \\
\quad-t_{2}^{1-\alpha} \int_{0}^{1} G\left(t_{2}, s\right) \phi_{q}\left(\int_{0}^{1} H(s, \tau) f(\tau, x(\tau)) d \tau\right) d s \mid \\
\leq \int_{0}^{1}\left|t_{1}^{1-\alpha} G\left(t_{1}, s\right)-t_{2}^{1-\alpha} G\left(t_{2}, s\right)\right| \phi_{q}\left(\int_{0}^{1}|H(s, \tau)| f(\tau, x(\tau)) \mid d \tau\right) d s \\
<\frac{\varepsilon}{\phi_{q}(M) \int_{0}^{1} \phi_{q}\left(\int_{0}^{1} H(s, \tau) d \tau\right) d s} \phi_{q}(M) \int_{0}^{1} \phi_{q}\left(\int_{0}^{1} H(s, \tau) d \tau\right) d s \\
=\varepsilon .
\end{aligned}
$$

So, $T$ is equicontinuous.

According to the Arzelà-Ascoli theorem, $T$ is a completely continuous operator. The proof is finished.

Define $P_{k}=\left\{x \in P:\|x\|_{1-\alpha}<r_{k}\right\}$ and then we define the related boundary $\partial P_{k}=\{x \in P$ : $\left.\|x\|_{1-\alpha}=r_{k}\right\}$ where $k$ is the positive integer.

Theorem 3.1 ([21] Krasnosel'skii fixed point theorem) Suppose E is a Banach space, and $P \subset E$ is a cone. Assume $P_{1}, P_{2}$ are two open and bounded subsets of $E$ coupled with $0 \in$ $P_{1}, \overline{P_{1}} \subset P_{2}$ and suppose $T: P \cap\left(\overline{P_{2}} \backslash P_{1}\right) \rightarrow P$ is a completely continuous operator such that one of the following conditions holds:

$\left(\mathrm{L}_{1}\right)\|T x\| \geq\|x\|$, if $x \in P \cap \partial P_{1}$, and $\|T x\| \leq\|x\|$, if $x \in P \cap \partial P_{2}$, 
$\left(\mathrm{L}_{2}\right)\|T x\| \leq\|x\|$, if $x \in P \cap \partial P_{1}$, and $\|T x\| \geq\|x\|$, if $x \in P \cap \partial P_{2}$.

Then $T$ has at least one fixed point in $P \cap\left(\overline{P_{2}} \backslash P_{1}\right)$.

Theorem 3.2 If there exist $\left.0<r_{1}<r_{2}, N_{1} \geq\left(\rho m \int_{0}^{1} \phi_{q}\left(\int_{0}^{1} H(s, \tau) d \tau\right) d s\right)\right)^{-1}, 0 \leq N_{2} \leq$ $\left.\left(n \int_{0}^{1} \phi_{q}\left(\int_{0}^{1} H(s, \tau) d \tau\right) d s\right)\right)^{-1}$ and $N_{1} r_{1}<N_{2} r_{2}$ such that the following conditions hold:

$\left(\mathrm{L}_{3}\right) f(t, x) \geq N_{1} r_{1},(t, x) \in[0,1] \times\left[0, r_{1}\right]$,

$\left(\mathrm{L}_{4}\right) f(t, x) \leq N_{2} r_{2},(t, x) \in[0,1] \times\left[0, r_{2}\right]$

then $T$ has at least one fixed point in $P \cap\left(\overline{P_{2}} \backslash P_{1}\right)$. In addition, $r_{1} \leq\|x\|_{1-\alpha} \leq r_{2}$.

Proof Step 1: Let $P_{1}=\left\{x \in P:\|x\|_{1-\alpha}<r_{1}\right\}$, by assumption $\left(\mathrm{L}_{3}\right)$, for all $t \in[0,1]$,

$$
\begin{aligned}
\|T x\|_{1-\alpha} & =t^{1-\alpha} \int_{0}^{1} G(t, s) \phi_{q}\left(\int_{0}^{1} H(s, \tau) f(\tau, x(\tau)) d \tau\right) d s \\
& \geq N_{1} r_{1} \int_{0}^{1} \rho m \phi_{q}\left(\int_{0}^{1} H(s, \tau) d \tau\right) d s \\
& \geq r_{1}=\|x\|_{1-\alpha} .
\end{aligned}
$$

Then we can get $\|T x\|_{1-\alpha} \geq\|x\|_{1-\alpha}\left(\forall x \in P \cap \partial P_{1}\right)$.

Step 2: Let $P_{2}=\left\{x \in P:\|x\|_{1-\alpha}<r_{2}\right\}$, by assumption $\left(\mathrm{L}_{4}\right)$, for all $t \in[0,1]$,

$$
\begin{aligned}
\|T x\|_{1-\alpha} & =t^{1-\alpha} \int_{0}^{1} G(t, s) \phi_{q}\left(\int_{0}^{1} H(s, \tau) f(\tau, x(\tau)) d \tau\right) d s \\
& \leq N_{2} r_{2} \int_{0}^{1} n \phi_{q}\left(\int_{0}^{1} H(s, \tau) d \tau\right) d s \\
& \leq r_{2}=\|x\|_{1-\alpha} .
\end{aligned}
$$

It is easy to obtain $\|T x\|_{1-\alpha} \leq\|x\|_{1-\alpha}\left(\forall x \in P \cap \partial P_{2}\right)$.

To conclude, the above proof satisfies the condition $\left(\mathrm{L}_{1}\right)$ of Theorem 3.1, that is to say, $T$ has at least one fixed point in $P \cap\left(\overline{P_{2}} \backslash P_{1}\right)$. In addition, $r_{1} \leq\|x\|_{1-\alpha} \leq r_{2}$.

The proof is finished.

Theorem 3.3 ([22]) Suppose $E$ is a Banach space, and $P \subset E$ is a cone. Assume $P_{3}, P_{4}$ are two open and bounded subsets of $E$ and $T: \overline{P_{3}} \rightarrow P$ is a completely continuous operator. If $\|T x\|_{1-\alpha}<r_{3}$, for $x \in \partial P_{3}$ and $\|T x\|_{1-\alpha} \geq r_{4}$, for $x \in \partial P_{4}$ hold, then $T$ has at least two fixed points in $\overline{P_{3}}$, and

$$
0 \leq\left\|x_{1}\right\|_{1-\alpha}<r_{3}<\left\|x_{2}\right\|_{1-\alpha} \leq r_{4} .
$$

Theorem 3.4 If there exist $\left.0<r_{3}<r_{4}, 0 \leq N_{3}<\left(\rho m \int_{0}^{1} \phi_{q}\left(\int_{0}^{1} H(s, \tau) d \tau\right) d s\right)\right)^{-1}, N_{4} \geq$ ( $\left.\left.n \int_{0}^{1} \phi_{q}\left(\int_{0}^{1} H(s, \tau) d \tau\right) d s\right)\right)^{-1}$ such that the following conditions hold:

$\left(\mathrm{L}_{5}\right) f(t, x)<N_{3} r_{3}, x \in \partial P_{3}$,

( $\left.\mathrm{L}_{6}\right) f(t, x) \geq N_{4} r_{4}, x \in \partial P_{4}$,

then $T$ has at least two fixed points in $\overline{P_{3}}$, and

$$
0 \leq\left\|x_{1}\right\|_{1-\alpha}<r_{3}<\left\|x_{2}\right\|_{1-\alpha} \leq r_{4} .
$$


Proof On the one hand, $P_{3}=\left\{x \in P:\|x\|_{1-\alpha}<r_{3}\right\}$, by assumption ( $\left.\mathrm{L}_{5}\right)$, for all $t \in[0,1]$,

$$
\begin{aligned}
\|T x\|_{1-\alpha} & =t^{1-\alpha} \int_{0}^{1} G(t, s) \phi_{q}\left(\int_{0}^{1} H(s, \tau) f(\tau, x(\tau)) d \tau\right) d s \\
& \leq N_{3} r_{3} \int_{0}^{1} n \phi_{q}\left(\int_{0}^{1} H(s, \tau) d \tau\right) d s \\
& <r_{3} .
\end{aligned}
$$

Then we can get $\|T x\|_{1-\alpha}<r_{3}\left(\forall x \in \partial P_{3}\right)$.

On the other hand, $P_{4}=\left\{x \in P:\|x\|_{1-\alpha}<r_{4}\right\}$, by assumption $\left(\mathrm{L}_{6}\right)$, for all $t \in[0,1]$,

$$
\begin{aligned}
\|T x\|_{1-\alpha} & =t^{1-\alpha} \int_{0}^{1} G(t, s) \phi_{q}\left(\int_{0}^{1} H(s, \tau) f(\tau, x(\tau)) d \tau\right) d s \\
& \geq N_{4} r_{4} \int_{0}^{1} \rho m \phi_{q}\left(\int_{0}^{1} H(s, \tau) d \tau\right) d s \\
& \geq r_{4} .
\end{aligned}
$$

Obviously, $\|T x\|_{1-\alpha} \geq r_{4}\left(\forall x \in \partial P_{4}\right)$ holds.

To conclude, the above proof satisfies the condition of Corollary 3.3, that is to say, $T$ has at least two fixed points in $\overline{P_{3}}$, which satisfy

$$
0 \leq\left\|x_{1}\right\|_{1-\alpha}<r_{3}<\left\|x_{2}\right\|_{1-\alpha} \leq r_{4}
$$

The proof is finished.

Suppose $\varphi, \psi$ are non-negative continuous convex functionals on $P, \theta$ is a non-negative continuous concave functional on $P$, and $\eta$ is a non-negative continuous functional on $P$. Define the following convex set:

$$
\begin{aligned}
& P(\varphi ; d)=\{x \in P: \varphi(x)<d\}, \\
& P(\varphi, \theta ; b, d)=\{x \in P: b \leq \theta(x), \varphi(x) \leq d\}, \\
& P(\varphi, \psi, \theta ; b, c, d)=\{x \in P: b \leq \theta(x), \psi(x) \leq c, \varphi(x) \leq d\},
\end{aligned}
$$

and a closed set

$$
P(\varphi, \eta ; a, d)=\{x \in P: a \leq \theta(x), \varphi(x) \leq d\}
$$

where $a, b, c, d$ are positive constants.

Theorem 3.5 ([23] Avery-Peterson fixed point theorem) Suppose P is a cone on the real Banach space E. Suppose $\varphi, \psi$ are non-negative continuous convex functionals on $P, \theta$ is a non-negative continuous concave functional on $P$, and $\eta$ is a non-negative continuous functional on P satisfying $\eta(\rho x) \leq \rho \eta(x)$ for $0 \leq \rho \leq 1$, for positive constants $k$ and $d$, we have

$$
\theta(x) \leq \eta(x), \quad\|x\| \leq k \varphi(x), \quad \forall x \in \overline{P(\varphi ; d)}
$$


Suppose $T: \overline{P(\varphi ; d)} \rightarrow \overline{P(\varphi ; d)}$ is a completely continuous operator and there exist $a, b, c>0$ with $a<b$ such that the following conditions hold:

$\left(\mathrm{H}_{1}\right)\{x \in P(\varphi, \psi, \theta ; b, c, d): \theta(x)>b\} \neq \emptyset$ and $\theta(x)>b$ for $x \in P(\varphi, \psi, \theta ; b, c, d)$,

$\left(\mathrm{H}_{2}\right) \theta(T x)>b$ for $x \in P(\varphi, \theta ; b, d)$ with $\eta(T x)>c$,

$\left(\mathrm{H}_{3}\right) \quad 0 \notin P(\varphi, \eta ; a, d)$ and $\eta(T x)<a$, for $P(\varphi, \eta ; a, d)$ with $\eta(x)=a$.

Then $T$ has at least three fixed points $x_{1}, x_{2}, x_{3} \in \overline{P(\varphi ; d)}$ such that

$$
\varphi\left(x_{i}\right) \leq d, \quad \theta\left(x_{1}\right)>b, \quad \theta\left(x_{2}\right)>a, \quad \eta\left(x_{2}\right)<b, \quad \eta\left(x_{3}\right)<a,
$$

where $i=1,2,3$.

Define the following functionals:

$$
\begin{aligned}
& \varphi(x)=\|x\|_{1-\alpha}, \quad \psi(x)=\eta(x)=\max _{t \in[0,1]}\left|t^{1-\alpha} x(t)\right|, \quad \theta(x)=\min _{t \in[0,1]}\left|t^{1-\alpha} x(t)\right|, \\
& N=\rho m \int_{0}^{1} \phi_{q}\left(\int_{0}^{1} H(s, \tau) f(\tau, x(\tau)) d \tau\right) d s,
\end{aligned}
$$

therefore, $\varphi, \psi, \eta, \theta$ satisfy the condition as mentioned above, and

$$
\rho \eta(x) \leq \theta(x) \leq \psi(x)=\eta(x), \quad\|x\|_{1-\alpha} \leq \varphi(x) .
$$

Theorem 3.6 Suppose $\left(\mathrm{H}_{0}\right)$ holds, there exist positive constants $a, b, c, d$ with $a<b<c=$ $b \rho^{-1}$,

$$
\begin{aligned}
& \left(\mathrm{H}_{4}\right) f(t, x) \leq \Gamma(\beta-1) \phi_{p}\left(d n^{-1}\right) \text { for }(t, x) \in[0,1] \times[0, d], \\
& \left(\mathrm{H}_{5}\right) f(t, x)>\phi_{p}\left(b N^{-1}\right) \text { for }(t, x) \in[0,1] \times[b, c], \\
& \left(\mathrm{H}_{6}\right) f(t, x)<\Gamma(\beta-1) \phi_{p}\left(a n^{-1}\right) \text { for }(t, x) \in[0,1] \times[0, a],
\end{aligned}
$$

Then $T$ has at least three fixed points $x_{1}, x_{2}, x_{3} \in \overline{P(\varphi ; d)}$ such that

$$
\varphi\left(x_{i}\right) \leq d, \quad \theta\left(x_{1}\right)>b, \quad \theta\left(x_{2}\right)>a, \quad \eta\left(x_{2}\right)<b, \quad \eta\left(x_{3}\right)<a,
$$

where $i=1,2,3$.

Proof For $x(t) \in \overline{P(\varphi ; d)}$, we have

$$
\varphi(x)=\|x\|_{1-\alpha} \leq d .
$$

According to $\left(\mathrm{H}_{4}\right)$, we have

$$
\begin{aligned}
\|T x(t)\| & =\left|t^{1-\alpha} \int_{0}^{1} G(t, s) \phi_{q}\left(\int_{0}^{1} H(s, \tau) f(\tau, x(\tau)) d \tau\right) d s\right| \\
& \leq \int_{0}^{1}\left|t^{1-\alpha} G(t, s)\right| \phi_{q}\left(\int_{0}^{1}|H(s, \tau)||f(\tau, x(\tau))| d \tau\right) d s \\
& <n \phi_{q}\left(\frac{\Gamma(\beta-1) \phi_{q}\left(\frac{d}{n}\right)}{\Gamma(\beta-1)}\right) \\
& =d .
\end{aligned}
$$


Hence, $\varphi(T x)=\|T x\|_{1-\alpha} \leq d$, that is to say, $T: \overline{P(\varphi ; d)} \rightarrow \overline{P(\varphi ; d)}$ is a completely continuous operator.

Let $x(t)=\frac{b}{\rho}, x(t) \in P(\varphi, \psi, \theta ; b, c, d)$ and $\theta\left(\frac{b}{\rho}\right)>b$, and so $\left\{x(t) \in P(\varphi, \psi, \theta ; b, c, d): \theta\left(\frac{b}{\rho}\right)>\right.$ $b\} \neq \emptyset$.

For $x(t) \in P(\varphi, \psi, \theta ; b, c, d)$, we obtain $b \leq x(t) \leq c=\frac{b}{\rho}$, then

$$
\begin{aligned}
\theta(T x) & =\min _{t \in[0,1]}\left|t^{1-\alpha} T x(t)\right| \\
& =\min _{t \in[0,1]} t^{1-\alpha} \int_{0}^{1} G(t, s) \phi_{q}\left(\int_{0}^{1} H(s, \tau) f(\tau, x(\tau)) d \tau\right) d s \\
& \geq \int_{0}^{1} \rho \max _{t \in[0,1]} t^{1-\alpha} G(t, s) \phi_{q}\left(\int_{0}^{1} H(s, \tau) f(\tau, x(\tau)) d \tau\right) d s \\
& >\rho m \int_{0}^{1} \phi_{q}\left(\int_{0}^{1} H(s, \tau) f(\tau, x(\tau)) d \tau\right) d s \frac{b}{N} \\
& =b .
\end{aligned}
$$

Hence, $\theta(T x)>b$ for $x \in P(\varphi, \psi, \theta ; b, c, d)$. That is to say, $\left(\mathrm{H}_{1}\right)$ holds.

Let $x \in P(\varphi, \theta ; b, d)$ with $\psi(T x)>c=\frac{b}{\rho}$, we obtain

$$
\theta(T x) \geq \rho \psi(T x)>\rho c=\rho \frac{b}{\rho}=b .
$$

Therefore, $\left(\mathrm{H}_{2}\right)$ is satisfied.

By $\theta(0)=0<a$, we have $0 \notin P(\varphi, \eta ; a, d)$.

Let $x \in P(\varphi, \eta ; a, d)$ and $\eta(x)=a$, we obtain $\varphi(x) \leq d$, according to $\left(\mathrm{H}_{6}\right)$,

$$
\begin{aligned}
\eta(T x) & =\max _{t \in[0,1]}\left|t^{1-\alpha} T x(t)\right| \\
& =\max _{t \in[0,1]} t^{1-\alpha} \int_{0}^{1} G(t, s) \phi_{q}\left(\int_{0}^{1} H(s, \tau) f(\tau, x(\tau)) d \tau\right) d s \\
& \leq n \int_{0}^{1} \phi_{q}\left(\int_{0}^{1} H(s, \tau) f(\tau, x(\tau)) d \tau\right) d s \\
& <n \phi_{q}\left(\frac{\Gamma(\beta-1) \phi_{q}\left(\frac{a}{n}\right)}{\Gamma(\beta-1)}\right) \\
& =a .
\end{aligned}
$$

Hence, the condition $\left(\mathrm{H}_{3}\right)$ holds.

The proof is finished.

\section{Illustrative example}

Consider the following nonlinear fractional differential equations:

$$
\left\{\begin{array}{l}
-D_{0^{+}}^{1.7}\left(\phi_{3.5}\left(D_{0^{+}}^{0.5} x(t)-3 x(t)\right)\right)=f(t, x(t)), \quad t \in(0,1], \\
\lim _{t \rightarrow 0^{+}} t^{0.5} x(t)=\sum_{k=1}^{\infty} \frac{1}{2^{2 k}(k-1) !} x\left(\frac{1}{2^{2 k}}\right), \\
\lim _{t \rightarrow 0^{+}} t^{0.3}\left(\phi_{3.5}\left(D_{0^{+}}^{0.5} x(t)-3 x(t)\right)\right)=\phi_{3.5}\left(D_{0^{+}}^{0.5} x(1)-3 x(1)\right)=0 .
\end{array}\right.
$$


Obviously, we choose $\alpha=0.5, \beta=1.7, p=3.5, \lambda=-3, \mu_{k}=\frac{1}{2^{2 k}(k-1) !}, \xi_{k}=\frac{1}{2^{2 k}}, \sum_{k=1}^{\infty} \mu_{k} \xi_{k}^{\alpha-1}=$ $\frac{e^{0.5}}{2}<1$. By simple calculation, we obtain $\rho \approx 0.04819, m \approx 0.0011246, n \approx 10.091$.

Part 1: In order to satisfy Theorem 3.1, by matlab, we get $N_{1}=3.1 \times 10^{4}, N_{2}=0.5, r_{1}=$ $0.2, r_{2}=4 \times 10^{4}$, so we know that the BVP (11) has at least one positive solution $x_{1}$ satisfy $0.2 \leq\left\|x_{1}\right\|_{1-\alpha} \leq 4 \times 10^{4}$, where

$$
f(t, x)=7 \times 10^{3} e^{t}+\sin x, \quad(t, x) \in[0,1] \times\left[0,4 \times 10^{4}\right]
$$

where $f(t, x)$ satisfies

(i) $f(t, x) \geq N_{1} r_{1}=6.2 \times 10^{3}$ for $(t, x) \in[0,1] \times[0,0.2]$,

(ii) $f(t, x)>N_{2} r_{2}=2 \times 10^{4}$ for $(t, x) \in[0,1] \times\left[0,4 \times 10^{4}\right]$.

Part 2: In order to make Theorem 3.3 hold, we choose $N_{3}=500, N_{4}=1, r_{3}=2, r_{4}=50$, then the BVP (11) has at least two positive solutions $x_{1}, x_{2}$, and

$$
0 \leq\left\|x_{1}\right\|_{1-\alpha}<2<\left\|x_{2}\right\|_{1-\alpha} \leq 50
$$

where

$$
f(t, x)=(t+5)^{3}+\frac{19}{4} x^{2}, \quad(t, x) \in[0,1] \times[0,100]
$$

where $f(t, x)$ satisfies

(i) $f(t, x)<N_{3} r_{3}=1000$ for $(t, x) \in[0,1] \times[0,2]$,

(ii) $f(t, x)>N_{4} r_{4}=50$ for $(t, x) \in[0,1] \times[0,50]$.

Part 3: In order to make Theorem 3.5 hold, we choose $a=0.75, b=2, c=b \rho^{-1}=$ $41.51, d=0.5$, then we know the BVP (11) has at least three positive solutions $x_{1}, x_{2}, x_{3}$ satisfying

$$
\begin{aligned}
& \|x\|_{0.5}\left(x_{i}\right) \leq 0.5 \quad(i=1,2,3), \quad \min _{t \in[0,1]}\left|t^{0.5} x_{1}\right|>2, \quad \min _{t \in[0,1]}\left|t^{0.5} x_{2}\right|>0.75, \\
& \max _{t \in[0,1]}\left|t^{0.5} x_{2}\right|<2, \quad \max _{t \in[0,1]}\left|t^{0.5} x_{3}\right|<0.75
\end{aligned}
$$

where

$$
f(t, x)=\frac{1}{10^{3}} \sqrt{t}+x^{13}, \quad(t, x) \in[0,1] \times[0,200],
$$

where $f(t, x)$ satisfies

(i) $f(t, x)<\Gamma(\beta-1) \phi_{p}\left(d n^{-1}\right)=0.617$ for $(t, x) \in[0,1] \times[0,0.5]$,

(ii) $f(t, x)>\phi_{p}\left(b N^{-1}\right)=3189.505$ for $(t, x) \in[0,1] \times[2,41.51]$,

(iii) $f(t, x)<\Gamma(\beta-1) \phi_{p}\left(a n^{-1}\right)=0.0342$ for $(t, x) \in[0,1] \times[0,0.75]$.

\section{Conclusions}

In this paper, we consider a class of nonlinear fractional differential equations coupled with the p-Laplacian operator and infinite-point boundary value conditions by means of the properties of Green's function, Krasnosel'skii fixed point theorem, the corollary of Krasnosel'skii fixed point theorem and the Avery-Peterson fixed point theorem. On top of that, we establish the suitable criteria to warrant the existence of the positive solutions 
for the BVP we learned. To the best of our knowledge, few researchers studied fractional differential equations with p-Laplacian by the Riemann-Liouville derivative, and this condition coupled with values at an infinite number of points is not covered in the previous situations; last but not least, it is more general for the fractional differential equation models.

\section{Acknowledgements}

The authors do appreciate the reviewers for careful reading and suggestions.

Funding

This work is supported by the National Nature Science Foundation of China (11271154).

Availability of data and materials

Not applicable.

\section{Competing interests}

The authors declare that they have no competing interests.

\section{Authors' contributions}

All authors contributed to proving the results and writing this paper. All authors read and approved the final manuscript.

\section{Publisher's Note}

Springer Nature remains neutral with regard to jurisdictional claims in published maps and institutional affiliations.

Received: 10 July 2018 Accepted: 12 November 2018 Published online: 20 November 2018

\section{References}

1. Venturi, D.: The numerical approximation of nonlinear functionals and functional differential equations. Phys. Rep. 732, 1-102 (2018)

2. Raissi, M., Karniadakis, G.E.: Hidden physics models: machine learning of nonlinear partial differential equations. J. Comput. Phys. 357, 125-141 (2017)

3. Wang, Q., Wen, J., Qiu, S., et al.: Numerical oscillations for first-order nonlinear delay differential equations in a hematopoiesis model. Adv. Differ. Equ. 1, 163 (2013)

4. Jiang, Y.F., Xia, B., Zhao, X., et al.: Data-based fractional differential models for non-linear dynamic modeling of a lithium-ion battery. Energy 135, 171-181 (2017)

5. Prakash, B., Setia, A., Bose, S.: Numerical solution for a system of fractional differential equations with applications in fluid dynamics and chemical engineering. Int. J. Chem. React. Eng. 15, 1-29 (2017)

6. Sopasakis, P., Sarimveis, H., Macheras, P., et al.: Fractional calculus in pharmacokinetics. J. Pharmacokinet. Pharmacodyn. 45, 1-19 (2017)

7. Zhang, L.H., Ahmad, B., Wang, G.T.: Monotone iterative method for a class of nonlinear fractional differential equations on unbounded domains in Banach spaces. Filomat 31, 1331-1338 (2017)

8. Wang, G.T., Pei, K., Agarwal, R.P., Zhang, L.H., Ahmad, B.: Nonlocal Hadamard fractional boundary value problem with Hadamard integral and discrete boundary conditions on a half-line. J. Comput. Appl. Math. 343, 230-239 (2018)

9. Guo, L.M., Liu, L.S., Wu, Y.H.: Existence of positive solutions for singular fractional differential equations with infinite-point boundary conditions. Nonlinear Anal., Model. Control 21, 635-650 (2016)

10. Bian, L.H., He, X.P., Sun, H.R.: Multiple positive solutions of m-point BVPs for third-order p-Laplacian dynamic equations on time scales. Adv. Differ. Equ. 2009, 262857 (2009)

11. Zhang, L.C., Zhang, W.G., Liu, X.P., Jia, M.: Existence of positive solutions for integral boundary value problems of fractional differential equations with p-Laplacian. Adv. Differ. Equ. 2017, 36 (2017)

12. Liu, X.P., Jia, M.: The method of lower and upper solutions for the general boundary value problems of fractional differential equations with p-Laplacian. Adv. Differ. Equ. 2017, 28 (2018)

13. Liu, X.P., Jia, M., Ge, W.G.: The method of lower and upper solutions for mixed fractional four-point boundary value problem with p-Laplacian operator. Appl. Math. Lett. 65, 56-62 (2017)

14. Oldham, K.B., Spanier, J.: The Fractional Calculus: Theory and Applications of Differentiation and Integration to Arbitrary Order. Mathematics in Science and Engineering. Academic Press, New York (1974)

15. Agarwal, R.P.: A propos d'une note de M. Pierre Humbert. C. R. Acad. Sci. 236, 2031-2032 (1953)

16. Weitzner, H., Zaslavsky, G.M.: Some applications of fractional equations. Commun. Nonlinear Sci. Numer. Simul. 8 273-281 (2003)

17. Bai, Z.B., Lü, H.S.: Positive solutions for boundary value problem of nonlinear fractional differential equation. J. Math. Anal. Appl. 311, 495-505 (2005)

18. Cabada, A., Kisela, T.: Existence of positive periodic solutions of some nonlinear fractional differential equations Commun. Nonlinear Sci. Numer. Simul. 50, 51-67 (2017)

19. Kilbas, A.A., Srivastava, H.M., Trujillo, J.J.: Theory and Applications of Fractional Differential Equations. Elsevier, New York (2006)

20. Xu, X.J., Jiang, D.Q., Yuan, C.J.: Multiple positive solutions for the boundary value problem of a nonlinear fractional differential equation. Nonlinear Anal., Theory Methods Appl. 71, 4676-4688 (2009)

21. Smart, D.R.: Fixed Point Theorems. Cambridge University Press, Cambridge (1974) 
22. Bai, Z.B.: Theory and Application of Boundary Value Problems for Fractional Differential Equations. China Sci. \& Technol. Press, Beijing (2013)

23. Avery, R.I., Peteson, A.C.: Three positive fixed points of nonlinear operators on ordered Banach spaces. Comput. Math. Appl. 42, 313-322 (2001)

Submit your manuscript to a SpringerOpen ${ }^{\mathcal{O}}$ journal and benefit from:

- Convenient online submission

$\checkmark$ Rigorous peer review

- Open access: articles freely available online

- High visibility within the field

- Retaining the copyright to your article

Submit your next manuscript at $\gg$ springeropen.com 